\title{
Quelque chose ... de remarquable in English-French acquisition: mandatory, informationally encapsulated computations in second language interpretation
}

\author{
Laurent Dekydtspotter and Jon C. Hathorn Indiana \\ University
}

We discuss the results of an experiment that investigates EnglishFrench learners' interpretation of quantifiers with detachable restrictions. Such quantifiers are ungrammatical in English. We investigate aspects of interpretation that rely on a highly idiosyncratic interface between grammar and general principles of conversational cooperation in native French. We show that a learning-theoretic challenge of the most severe kind arises in English-French acquisition unless second language acquisition is constrained by very specific relations between syntactic, semantic and pragmatic modules. We therefore argue that the emergence of knowledge of interpretation in English-French interlanguage suggests mandatory, informationally encapsulated computations. This supports Schwartz's $(1986 ; 1987 ; 1999)$ contention that interlanguage knowledge is constrained by a mental organization in which Universal Grammar provides the contents of a largely universal processor devoted to language.

\section{A severe poverty of the stimulus problem}

In French, unlike in English, the existential quantifier quelque chose 'something' may take an adjectival restriction introduced by the particle $d e$. 
This adjectival restriction may appear together with the quantifier as in (1) or may be separated from it as in (2) (Huot, 1981; Azoulay-Vicente, 1985; Birdsong, 1985; Hulk and Verheugd, 1994).

1) Quelque chose de remarquable a été observé par chacun des chercheurs. something of remarkable was observed by each of-the researchers 'Something remarkable was observed by each of the researchers.'

2) Quelque chose a été observé de remarquable par chacun des chercheurs. something was observed of remarkable by each of-the researchers 'Something remarkable was observed by each of the researchers.'

The permutation of the continuous quantifier in (1) into the discontinuous quantifier in (2) is accompanied by very subtle interpretive effects of a pragmatic nature. As a French speaker in a speech situation, if you hear the continuous sentence in (1), a range of situations seems possible: It could be that all the researchers witnessed a different remarkable object or that all the researchers witnessed the same remarkable object, or any case in between. In contrast, out of the blue, the discontinuous sentence in (2) implies that the researchers did not observe the same thing. This implication can, however, be defeated. That is to say, one can assert (2) without taking it as true in the context that the researchers did not observe the same thing. Therefore, (2) may be followed by (3), which denies the implication, without the resulting discourse being contradictory.

3) En fait, la même chose a été observée par tous les chercheurs. in fact the same thing was observed by all the researchers 'In fact, the same thing was observed by all the researchers.'

The implication that the researchers did not observe the same thing is, hence, not part of the literal semantics, but arises as a pragmatic implicature. The paradigm of situations and syntactic structures (with the implicature taken as true) is presented in Table 1.

A (second) language acquirer must come to know that the discontinuous sentence (2) a priori excludes situations where the same object was observed by all researchers. However, there are serious obstacles in the path of the learner. If the learner extends the interpretation of the familiar continuous quantifier structure in (1) to the unfamiliar discontinuous quantifier structure in (2), no evidence will ever inform the learner that the discontinuous quantifier structure in (2) is not interpretively a variant of the continuous quantifier structure in (1). This is 
Table 1 Interpretive asymmetries in continuous and discontinuous $\mathrm{Q}$ de $\mathrm{A}$ sentences

\begin{tabular}{lll}
\hline Situations of use & $\begin{array}{l}\text { Continuous Q de A } \\
\text { sentence }\end{array}$ & $\begin{array}{l}\text { Discontinuous Q de A } \\
\text { sentence }\end{array}$ \\
\hline $\begin{array}{c}\text { Different object(s) } \\
\text { per researcher }\end{array}$ & Yes & Yes \\
$\begin{array}{c}\text { The same object(s) } \\
\text { for all researchers }\end{array}$ & Yes & No \\
\hline
\end{tabular}

because in every situation where a discontinuous quantifier structure as in (2) is used, the continuous quantifier structure as in (1) may be used as well. The learner thus never encounters a situation where only discontinuous quantifier structures can be used.

Even if the learner does not analogize discontinuous quantifier structures to continuous quantifier structures, severe problems remain. Situations of use do not unambiguously reflect the form-meaning relations in Table 1 . The implication that the same remarkable object was not observed by all researchers can be suspended by additionally uttering the corrective statement (3). Such corrective statements confuse form-meaning relations in actual use, because the learner will not reliably encounter the discontinuous quantifier structure in (2) only in situations where each researcher observed (at least) a different remarkable object. This is because the discontinuous sentence in (2) can be followed by sentence (3) which specifies that the same object was observed by all. Additionally, the corrective statement in (3) is also compatible with the continuous sentence in (1). Hence, corrective statements do not uniquely flag the interpretation of sentences with discontinuous quantifiers, since they can be found across the continuous/discontinuous dimension.

Thus, the highly unstable correspondences between sentence structure and situations make the likelihood of grasping the eccentric pattern in Table 1 exceedingly remote in actual use. Additionally, ambiguity in situations of use may come from the fact that the information known to the interlocutors is partial, so that the learner's information state may be compatible with a range of interpretations. In particular, one interlocutor could take a sentence as true on one interpretation, while the other interlocutor takes it as true on a different interpretation without recognizing 
that their interpretations are different. Partiality will prevent the learner from honing in on the interpretation in the general case.

In addition to problems in the character of the environment, learning problems also arise as a function of the learner. If learning proceeds inductively by making inferences that can be disconfirmed by further evidence, (erroneous) beliefs about the facts under discussion can cause unlearning at any point of acquisition. Induction, therefore, cannot guarantee that the relevant grammatical properties will arise in the general case. Hence, if such properties arise predictably in the course of development, then these properties are more feasibly viewed as an expression of a specific mental organization inherent in the learner.

Further, we note that the discontinuous structure in (2) is subject to stylistic constraints that make it unlikely to be part of the daily exposure in relatively early classroom acquisition. This adds to the complexities facing inductive acquisition in actual situations of use. In the context of (adult) English-French acquisition where instruction might play a role, it is also important to note that the construction and its interpretation are not the topic of instruction, and that native speakers are not consciously aware of the grammatical properties under discussion.

\section{Scalar implicatures: syntax, semantics and pragmatics}

The interpretation of discontinuous sentences seems to require semantically constrained computations beyond syntax-semantics mappings. To see this, we consider the semantic representations resulting from scope interactions among the quantifiers and their truth conditions. Both continuous (1) and discontinuous (2) introduce an existential quantifier quelque chose 'something' (analysed with existential $\exists$ ) and a universal quantifier chacun des chercheurs 'everyone of the researchers' (analysed with universal $\forall$ ). These quantifiers bind variables in the theta positions of the verb's semantic representation and interact with one another. The existential quantifier quelque chose takes scope either under the universal quantifier chacun des chercheurs as in the $\forall \exists$ representation (4) or over it as in the $\exists \forall$ representation (5). Abstracting away from tense, the representations in (4) and (5) thus constitute the range of scope-dependent semantic representations potentially available to (1) and (2). 
4) $\forall y[\operatorname{researcher}(y) \rightarrow[\exists x[\operatorname{thing}(x) \wedge \operatorname{remarkable}(x) \wedge \operatorname{observe}(x)(y)]]]$ 'For every researcher there is a remarkable thing such that he/she observed it.'

5) $\exists x[\operatorname{thing}(x) \wedge \operatorname{remarkable}(x) \wedge \forall y[\operatorname{researcher}(y) \rightarrow[\operatorname{observe}(x)(y)]]]$ 'There is a remarkable thing such that every researcher observed it.'

The semantic differences between (4) and (5) reside in the scope of the quantifiers. In (4) quelque chose takes scope under the universal quantifier chacun des chercheurs. In (5) quelque chose takes scope over the universal quantifier chacun des chercheurs. On the $\forall \exists$ representation in (4), the sentence is true of (past) situations where each researcher is such that some remarkable thing was observed (but not necessarily the same object). On the $\exists \forall$ representation in (5), the sentence is true of (past) situations where some (unique) remarkable thing is such that every researcher observed it. Thus, (4) denotes the set of (past) situations where for every researcher there is a remarkable object that he or she observed. The representation in (5) denotes the set of (past) situations where for some (unique) remarkable object it is the case that every researcher observed it. Hence, the representations in (4) and (5) are both true of (past) situations where the same remarkable object was observed by every researcher, but (5) exclusively specifies such situations.

Semantically, the representations in (4) and (5) stand in an asymmetrical entailment relation: whenever (5) is true, (4) is true, but not the other way around. The entailment relation follows from the fact that the situations that make (5) true and the situations that make (4) true stand in the inclusion relation in (6): The set of situations that verify the representation (5) with the quantifiers in the $\exists \forall$ scope relation (i.e., the set of situations where the same remarkable object is observed by each researcher) is a subset of the set of situations that verify the representation (4) with the quantifiers in the $\forall \exists$ scope relation (i.e., the set of situations where each researcher observed some remarkable object, and possibly the same one).

6) $\{\mathrm{s} \mid \mathrm{s}$ is characterized by (5) $\} \subseteq\{\mathrm{s} \mid \mathrm{s}$ is characterized by (4) $\}$

Given that the $\forall \exists$ representation in (4) and $\exists \forall$ representation in (5) together constitute the space of possible semantic representations for both the continuous sentence in (1) and the discontinuous sentence in (2), the asymmetry between (1) and (2) in Table 1 is unexpected on the basis of possible semantic representations alone. This is because both (4) and (5) will be true in situations where the same remarkable object 
was observed by all the researchers, yet the sentences (1) and (2) are not equally acceptable in this context. Thus, the interpretive intuitions in Table 1 must require pragmatically induced computations beyond Logical Form (LF) representations, as we now show.

Since Grice (1975), language use is assumed to be governed by domain-general principles of conversational cooperation. In particular, the Maxim of Quantity (7) requires that the strongest statement consistent with what is known be made.

7) Maxim of Quantity: make your contribution as informative as required, and no more informative than is required. (Grice, 1975)

Horn $(1985 ; 1989 ; 1992)$ shows that informational strength relations play a significant role in determining how Grice's principles apply on specific domains such as quantification. Given the semantics of quantification, the $\exists \forall$ representation (5) asymmetrically entails the $\forall \exists$ representation (4). This asymmetric entailment creates an informational scale in which the entailing ('subset') representation (5) is informationally stronger than the entailed ('superset') $\forall \exists$ representation (4). The $\exists \forall$ representation (5) uniquely characterizes the speech context, whereas the $\forall \exists$ representation (4) allows for other possibilities. Given that (5) is informationally stronger than (4), the Maxim of Quantity (7) would seem to require that in a context where the $\exists \forall$ representation (5) is true, (5) - as the more informative statement - should be the LF representation. Conversely, on the assumption that the Maxim of Quantity is satisfied, the $\forall \exists$ representation (4) at the LF-interface should trigger the implicature that the stronger statement (5) is not verified. In other words, if you could have made the stronger statement (5), you would have. Since you did not make the stronger statement, it must not be true.

We now turn to the manner in which the word order asymmetries arise in French. First, an idiosyncratic syntax-semantics (LF-interface) mapping is required. The fact that discontinuous (2) a priori describes a situation where each researcher observed a different object requires the $\forall \exists$ representation (4), because (4) is the only representation that allows for such situations. Following de Swart's work (1992) on constructions with discontinuous constituents, we adopt the interface constraint in (8).

8) In a construction: $\mathrm{Q}_{2 \mathrm{j}} \ldots \mathrm{Q}_{1} \ldots\left[\mathrm{e}_{\mathrm{j}}\right.$ [restriction of $\left.\left.\mathrm{Q}_{2}\right]\right] \ldots \mathrm{Q}_{2}$ cannot take scope over $\mathrm{Q}_{1}$. Semantic formulation: A quantifier $\mathrm{Q}_{1}$ can only separate a quantifier $\mathrm{Q}_{2}$ from its restrictive clause if $\mathrm{Q}_{1}$ has wide scope (or is scopally independent from $\mathrm{Q}_{2}$ ). 
The constraint in (8) fixes the scope of a discontinuous quantifier $\left(\mathrm{Q}_{2}\right)$ in terms of the placement of its restriction with respect to some other scope-bearing element. The syntactic constraint in (8) is necessary but not sufficient to account for the pattern in Table 1. This is because the $\forall \exists$ representation in (4), forced by the constraint in (8), is true not only of situations where each researcher observed a potentially different object, but also of situations where it turns out that the same object was observed. The mapping in (8) is, therefore, necessarily accompanied by a pragmatic inference. Hence, (8) must be supplemented by Gricean interpretive computations that lead to the exclusion of the set of situations described by (5).

In sum, the interpretive differences between (1) and (2) follow if:

- the sentence (1) maps to the $\forall \exists$ semantic representation in (4) or the $\exists \forall$ semantic representation in (5) at the LF-interface, whereas sentence (2) maps uniquely to the $\forall \exists$ representation (4) at the LF interface; and - a scalar implicature "(5) does not hold" is induced by the $\forall \exists$ representation (4) at LF, satisfying the Gricean Maxim of Quantity through a grammatically provided semantic scale.

In other words, the pattern in Table 1 arises because a grammatically calculated scalar implicature supervenes on the syntax-linked LFrepresentation (4) of the discontinuous sentence in (2) in order to satisfy principles of conversational cooperation. The continuous sentence in (1) receives the $\forall \exists$ representation in (4) or the $\exists \forall$ representation in (5), whereas the discontinuous sentence in (2) receives the $\forall \exists$ representation in (4) at LF by virtue of the constraint in (8). The representation in (4) at the LF-interface pragmatically implies "and not (5)" by the Maxim of Quantity (Grice, 1975) in a grammatically provided, entailment-based informational scale. In the absence of the computation of an implicature as part of interpretive knowledge, no asymmetry in the interpretation of (1) and (2) could arise. If an asymmetry arises, it is because an implicature is mandatorily calculated.

The derivation of scalar implicatures described above crucially relies on asymmetric scope relations. There are other asymmetries that support the idiosyncratic derivation of a scope-induced implicature through a grammatically provided informational scale. Only universal 
quantifiers (such as toujours 'always') trigger the implicature, yielding an asymmetry between (9a) and (9b). Existential quantifiers (such as parfois 'sometimes') do not yield such an asymmetry between (10a) and (10b).

9) a. Quelque chose a toujours été observé de remarquable le soir. something has always been observed of remarkable evenings. 'Something remarkable was always observed in the evenings.'

b. Quelque chose de remarquable a toujours été observé le soir. something of remarkable has always been observed evenings. 'Something remarkable was always observed in the evenings.'

10) a. Quelque chose a parfois été observé de remarquable le soir. something has sometimes been observed of remarkable evenings. 'Something remarkable was sometimes observed in the evenings.'

b. Quelque chose de remarquable a parfois été observé le soir. something of remarkable has sometimes been observed evenings. 'Something remarkable was sometimes observed in the evenings.'

With the universal event quantifier toujours, an implicature is generated by virtue of the semantics of the scopal interactions between the universal quantifier toujours and the existential quantifier quelque chose. In (9), the alternative scopes have truth-conditional import, so that the situations corresponding to the stronger $\exists \forall$ representation are excluded under the Gricean Maxim of Quantity, given the $\forall \exists$ representation at LF. Thus (9a) and (9b) subtly contrast in the relative availability of the same object reading (out of the blue). That is to say, if you know that the same remarkable object is always observed, then (9b) is a better characterization than (9a), although again (9a) is not an impossible statement, as discussed above. The contrast in (9) follows if the discontinuous syntax requires the $\forall \exists$ representation at LF, and if the LF $\forall \exists$ representation triggers an implicature that the $\exists \forall$ representation is not verified.

With the existential quantifiers parfois and quelque chose, there is no truth conditional import of the relative scopes of quelque chose and parfois: The representations map to the same set of situations on either scope of parfois with respect to quelque chose. Because scope relations do not lead to asymmetric truth conditions in (10), no implicature is generated. Thus, if you think of the same object per relevant observation, both (10a) and (10b) are possible characterizations out of the blue. The asymmetry that exists between (9a) and (9b) does not exist between (10a) and (10b). Hence, the implicatures that concern us here 
are crucially computed through scales determined by asymmetric entailment relations. We return to the importance of computations through scales in a discussion of informational encapsulation (see section IV).

\section{A second language acquisition experiment}

\section{Methods}

Our experiment investigated English-French learners' acceptance of continuous and discontinuous sentences in situations where there was one and the same object for all human participants vs. situations where there were different objects per human participant. Our respondents included a group of 40 adult classroom learners of French enrolled in the third semester of the basic language program at Indiana University. Our respondents also included a group of 20 students enrolled in sixth semester French language proficiency courses at Indiana University. Magnan (1986) found that most English-speaking students having completed one year of university study are concentrated at the intermediate low/mid levels in French language proficiency on the ACTFL (American Council of the Teaching of Foreign Languages) scale. Those having completed two and one half years are concentrated on the intermediate high level on the ACTFL scale. Thus, we refer to the 40 classroom learners of French in third semester French as the low/mid intermediate group, and we refer to the 20 classroom learners of French in the sixth semester of French language proficiency as the high intermediate group. The respondents provided background information on their native language, on their exposure to French and on any other languages they had studied or acquired. We excluded from consideration volunteers whose first language (L1) was not English. Our native comparison group included (adult) native French students, who were like our experimental groups: linguistically naive young adults. These native French speakers were completing coursework for the Bachelor of Arts (Licence) degree in English at the Universite de Lille in France. They were enrolled in English language proficiency in their third year of university studies. This group provides evidence of the ability of our instrument to capture reflexes of mental organization in a group of respondents whose knowledge of French is not in dispute. 
Experimental items consisted of eight sets of quadruples. Each quadruple included continuous vs. discontinuous sentences crossed with stories involving a different object or the same object for all human participants. The request for information was a constant. The lexical material within a given quadruple was identical. It was limited to vocabulary familiar to respondents even at the most elementary level investigated. Thus, each narrative (one providing a scenario with a different object, and one providing the same object for all human participants) appeared twice: once paired with a sentence exemplifying a continuous sentence in response to the request for information and once paired with a sentence exemplifying a discontinuous sentence in response to the same request for information. Eighteen fillers were blended in with the rest of the task to offset the expected imbalance between the positive and negative responses, yielding a total of 50 items. The 50 items were presented in three distinct random orders.

Respondents were told that they were judges for an (imaginary) comprehension exercise in Madame Goyette's English class. As judges, respondents were presented with excerpts from the transcripts of the comprehension exercise. Each experimental item consisted of a story told in English to French pupils by a character, named Mrs Briggs. When the story was being told, the pupils' teacher, Madame Goyette, was not in the room. After the story had been told, Madame Goyette prompted the pupils with a question in French about the story that Mrs Briggs told: Qu'est-ce qui s'est donc passé? ('So, what happened?'). Following this question, a pupil's answer in response to Madame Goyette's information request was provided to the respondents. Respondents indicated whether the answer was an adequate response to the information query. The respondents were told that an adequate response need not be complete and that it need not have all the details of the relevant aspects of the story. ${ }^{1}$ It merely should not mislead

\footnotetext{
${ }^{1}$ It was suggested to us by anonymous Second Language Research reviewers that the term 'adequate' risks potential misinterpretations by respondents, or that our approach would appeal the most to respondents already sensitized to these subtle distinctions by their training. Our respondents were randomly chosen from a population of French classroom learners. Although the subjects were not chosen for their linguistic sophistication in the relevant area, when we administered the practice examples, we found that they could easily determine that a sentence compatible with the facts of the scenarios would nevertheless be misleading to one who had only that sentence to go on, and that they could accept or reject answers on that basis.
} 
the hearer about the facts. Examples sensitized the respondents to the types of inferences that would mislead a hearer. The following examples illustrate the test methodology. (The full list of sentences is provided in Appendix 1.)

Sample Test Item 1: Different objects with continuous answer

Mrs Briggs: Having landed on the planet Omega, the three scientists of the interstellar expedition separated to begin their exploration of the planet. The first scientist observed a new life form. The second scientist observed the fast growth of interesting crystals. The third scientist looked up from his notebook just in time to observe a meteor crash into the surface of the planet.

Mme Goyette: Qu'est-ce qui s'est donc passé?

Élève: Quelque chose-de remarquable - a été observé par chacun des scientifiques.

IS THIS AN ADEQUATE RESPONSE? YES NO CANNOT DECIDE

Sample Test Item 2: Different objects with discontinuous answer

Mrs Briggs: Having landed on the planet Omega, the three scientists of the interstellar expedition separated to begin their exploration of the planet. The first scientist observed a new life form. The second scientist observed the fast growth of interesting crystals. The third scientist looked up from his notebook just in time to observe a meteor crash into the surface of the planet.

Mme Goyette: Qu'est-ce qui s'est donc passé?

Élève: Quelque chose a été observé-de remarquable-par chacun des scientifiques.

IS THIS AN ADEQUATE RESPONSE? YES NO CANNOT DECIDE

The English narratives ensured that all respondents unambiguously understood the context in which the answer was uttered. This allowed us to study learners at low levels of proficiency, since such learners might not be able to deal with an entire context in the target language. It could be argued that using English might also detract from learner performance, since it requires switching from one mode of expression to another. However, in the study of knowledge of interpretive effects where understanding of the situation is crucial, the English scenarios nearly guarantee that the respondent is in the relevant information state.

As a final methodological note relevant to the interpretation of results, we note that any interpretive asymmetry of the type considered in Table 1 logically requires that an implicature mandatorily accompany the $\forall \exists$ LF representation. Unless the implicature is mandatorily 
generated, no asymmetry is expected at all, given the range of possible semantic representations at LF. In contradistinction, the lack of a detectable asymmetry does not demonstrate the absence of implicature. Only the presence of an asymmetry along the lines discussed above is revealing of learners' knowledge. We first consider the ability of our instrument to detect the pattern previously shown in Table 1 in a group of native speakers. We then discuss our English-French results.

\section{Results and discussion}

The results for our group of French native speakers in Table 2 reveal a relevant pattern of asymmetries: there is no significant difference between continuous answers and discontinuous answers in differentobject contexts $(t(15)=1.15, p=.267)$, but there is a crucial significant difference between continuous answers and discontinuous answers in same-object contexts $(t(15)=2.50, p=.025)$. The French native speakers' acceptance rates of continuous answers in same-object vs. differentobject scenarios are virtually flat $(t(15)=.09, p=.930)$. In contrast, acceptance rates of discontinuous answers in same-object versus different-object scenarios differ significantly $(t(15)=2.16, p=.048)$.

Crucially, the asymmetries in the response pattern in Table 2 cannot logically be accounted for unless implicatures are calculated. We note that if implicatures were not systematically calculated in accordance with Gricean cooperation maxims, no difference between the acceptance of answers in different-object vs. same-object contexts would be expected at all. This is because $\forall \exists$ representations hold true of both types of situations. Hence, a post-LF pragmatic calculation must supervene on $\forall \exists$ LF representations for there to be any effect whatsoever in same-object contexts.

Table 2 French native speakers $(n=16)$ : percentage 'yes' on continuous vs. discontinuous answers

\begin{tabular}{llllll}
\hline & \multicolumn{2}{l}{ Continuous answers } & & \multicolumn{2}{c}{ Discontinuous answers } \\
\cline { 2 - 3 } & Mean (\%) & SD & & Mean (\%) & SD \\
\hline Different objects & 82.81 & 26.17 & & 71.09 & 33.76 \\
The same object & 82.03 & 25.40 & & 61.72 & 32.43 \\
\hline
\end{tabular}


We now consider the English-French learners. $T$-tests were performed for each subgroup. We, therefore, adopt a Bonferroni protection level of $\alpha=.05 / 2=.025$. As shown in Table 3 , the low $/ \mathrm{mid}$ intermediate learners $(n=40)$ accepted continuous and discontinuous answers with scenarios involving a different object per participant at very similar rates $(t(39)=.70, p=.489)$. They also accepted continuous and discontinuous answers in the same-object condition at similar rates $(t(39)=.15, p=.881)$. Low $/ \mathrm{mid}$ intermediate learners were biased against accepting the answers in same-object context when compared to different-object contexts in both conditions: for continuous answers $(t(39)=3.97, p<.0005)$ and for discontinuous answers $(t(39)=3.11$, $p=.003)$.

Thus, no effect of word order was found for this population: low/mid intermediate learners interpreted continuous answers and discontinuous answers alike. However, low/mid intermediate learners accepted fewer answers in same-object contexts than in different-object contexts. Again, this contrast is unexpected purely in terms of the semantics of the possible LF representations involved. This is because the semantics predict that the value "true" is returned in same-object situations. Similar acceptance rates are expected in all four cells, contrary to what was found.

Given possible LF representations, the pattern in Table 3 follows only if intermediate low/mid English-French learners typically access $\forall \exists$ representations at LF but typically not $\exists \forall$ representations (see discussion below), and if the situations that correspond to the $\exists \forall$ scope configuration are pragmatically excluded when a $\forall \exists$ representation is derived at LF. Our first conclusion imposed by logical necessity given the range of LF representations is that the pattern of asymmetry found in low/mid English-French learners' interpretation is such that $\forall \exists$ representations at LF induce scalar implicatures in obligatory fashion.

Table 3 Low/mid intermediate learners $(n=40)$ : percentage 'yes' on continuous vs. discontinuous answers

\begin{tabular}{llllll}
\hline & \multicolumn{2}{l}{ Continuous answers } & & \multicolumn{2}{c}{ Discontinuous answers } \\
\cline { 2 - 3 } \cline { 6 - 6 } & Mean (\%) & SD & & Mean (\%) & SD \\
\hline Different objects & 76.25 & 21.52 & & 74.06 & 19.28 \\
The same object & 59.06 & 22.64 & & 59.69 & 22.91 \\
\hline
\end{tabular}


In contrast, high intermediate learners $(n=20)$ exhibit sensitivity to word order in interpretation; this is shown in Table 4. With continuous answers, acceptance rates are statistically flat in different-object and same-object contexts $(t(19)=.47, p=.645)$. With discontinuous answers, however, the acceptance rate in different-object contexts marginally contrasts with the acceptance rate in same-object contexts $(t(19)=2.32, p=.032)$. The acceptance rates for continuous and discontinuous answers in different-object contexts are completely flat $(t(19)=.00, p=1.00)$. But, crucially, the acceptance rates for continuous and discontinuous answers in same-object contexts contrast significantly $(t(19)=2.60, p=.017)$.

Differences between the two groups reveal an acquisition sequence, associated with the interpretation of continuous and discontinuous answers. An independent samples $t$-test comparing the relative acceptance of continuous answers versus discontinuous answers as descriptions of same-object scenarios confirms that the two groups are indeed statistically distinct $(t(58)=2.09, p=.041)$. The behaviour of high intermediate learners (contra low/mid intermediate learners) is not unlike the pattern produced by the French native speakers.

Thus, for the high intermediate and native groups, a statistically significant difference of means arose between continuous and discontinuous answers in same-object scenarios, but not with different-object scenarios. Given that the range of possible semantic representations cannot alone account for such an asymmetry, we prima facie conclude that high intermediate learners assigned $\forall \exists$ representations of the type in (4) to discontinuous sentences, and that they obligatorily computed implicatures of the type "and not (5)". The change between the low/mid intermediate learners and the high intermediate learners seems characterizable in terms of the ability of high intermediate learners to retreat

Table 4 High intermediate learners $(n=20)$ : percentage 'yes' on continuous vs. discontinuous answers

\begin{tabular}{llllll}
\hline & \multicolumn{2}{l}{ Continuous answers } & & \multicolumn{2}{c}{ Discontinuous answers } \\
\cline { 2 - 3 } & Mean (\%) & SD & & Mean (\%) & SD \\
\hline Different objects & 84.38 & 28.64 & & 84.38 & 21.41 \\
The same object & 80.63 & 17.43 & & 66.88 & 25.74 \\
\hline
\end{tabular}


from the over-exclusion of situations in same-object contexts with continuous answers only.

We discuss the distribution of individual responses by comparing for each individual the rate of acceptance of discontinuous answers in same-object contexts versus different-object contexts. This distribution is provided in Figure 1. On the axis of abscissa appear the relative measures of acceptance of discontinuous answers in same-object contexts vs. different-object contexts. This is obtained by subtracting the percentage 'yes' responses to discontinuous answers in same-object contexts from the percentage 'yes' responses to discontinuous answers in different-object contexts. A value above (to the right of) zero indicates a higher acceptance of discontinuous answers in different-object contexts vs. same-object contexts. On the axis of the ordinate appears the number of individuals. That there are few respondents falling on the negative side (left of zero) demonstrates a bias against accepting discontinuous answers in same-object contexts.

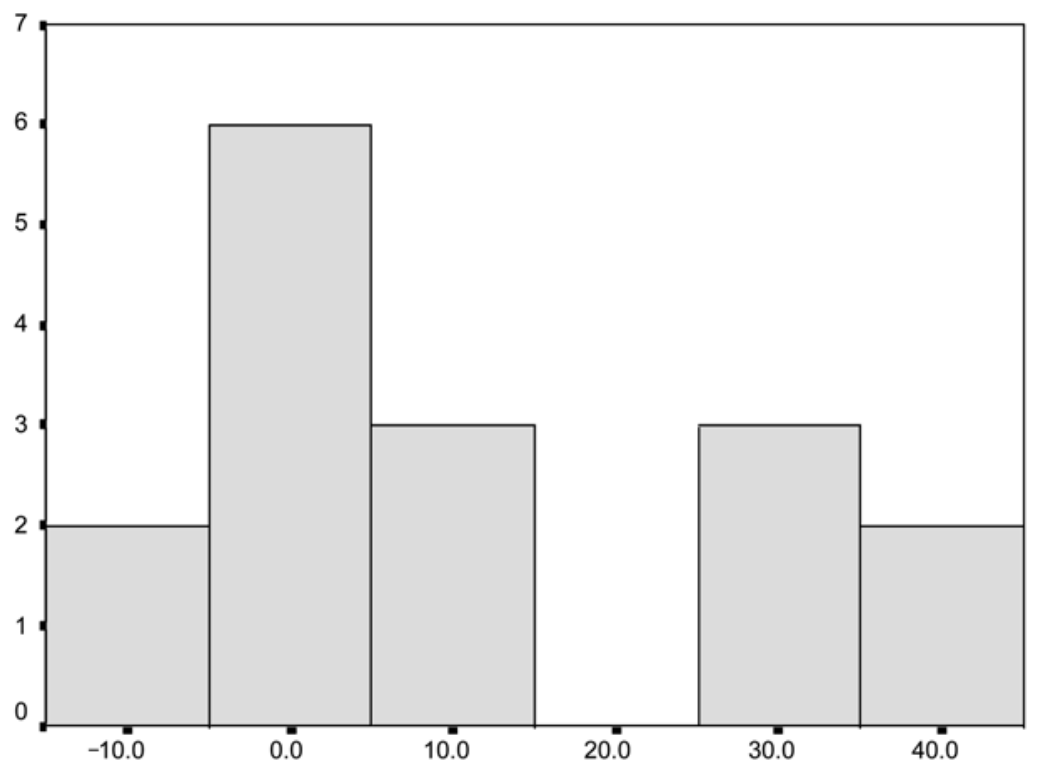

Figure 1 Native speakers $(n=16)$ : percentage 'yes' responses to discontinuous answers in different-object contexts minus percentage 'yes' responses to discontinuous answers in same-object contexts

Notes: Difference: accepted discontinuous answers in different vs. same-object contexts; $\mathrm{SD}=15.95 ;$ Mean $=10.2$. 
Similar patterns emerged in both learner populations as well. Individual responses showed a bias in the expected direction: There was a greater number of respondents accepting discontinuous answers in different-object contexts over same-object contexts than there were respondents accepting discontinuous answers in same-object contexts over different-object contexts. Figure 2 shows that an asymmetrical distribution was in evidence in the low/mid intermediate learners.

A strongly regimented pattern of behaviour was obvious in the individual distribution of the high intermediate learners, as shown in Figure 3. There was one individual with a marked response pattern in the opposite direction. However, this response pattern was in stark contrast with the rest of the respondents.

Hence, despite the range of factors that may affect performance, the pattern of individual responses in all three groups suggested that mental structure compels respondents to resist accepting discontinuous answers in same-object contexts.

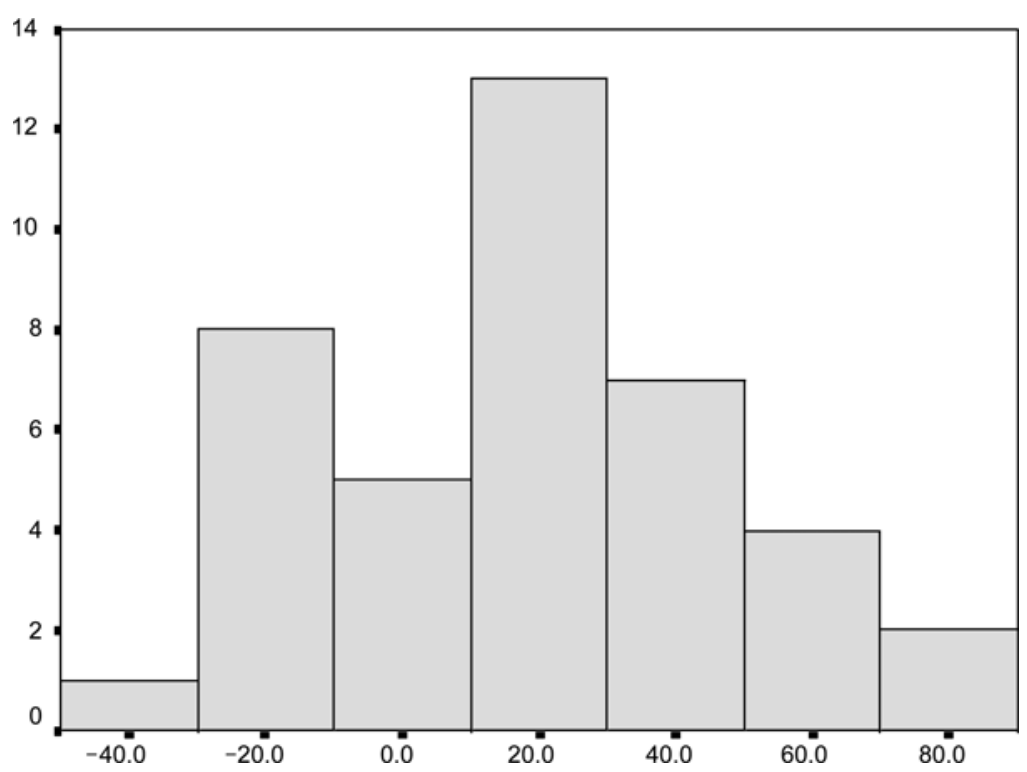

Figure 2 Low/mid intermediate learners $(n=40)$ : percentage 'yes' responses to discontinuous answers in different-object contexts minus percentage 'yes' responses to discontinuous answers in same-object contexts

Notes: Difference: accepted discontinuous answers in different vs. same-object contexts; $\mathrm{SD}=27.98$; Mean $=17.2$. 


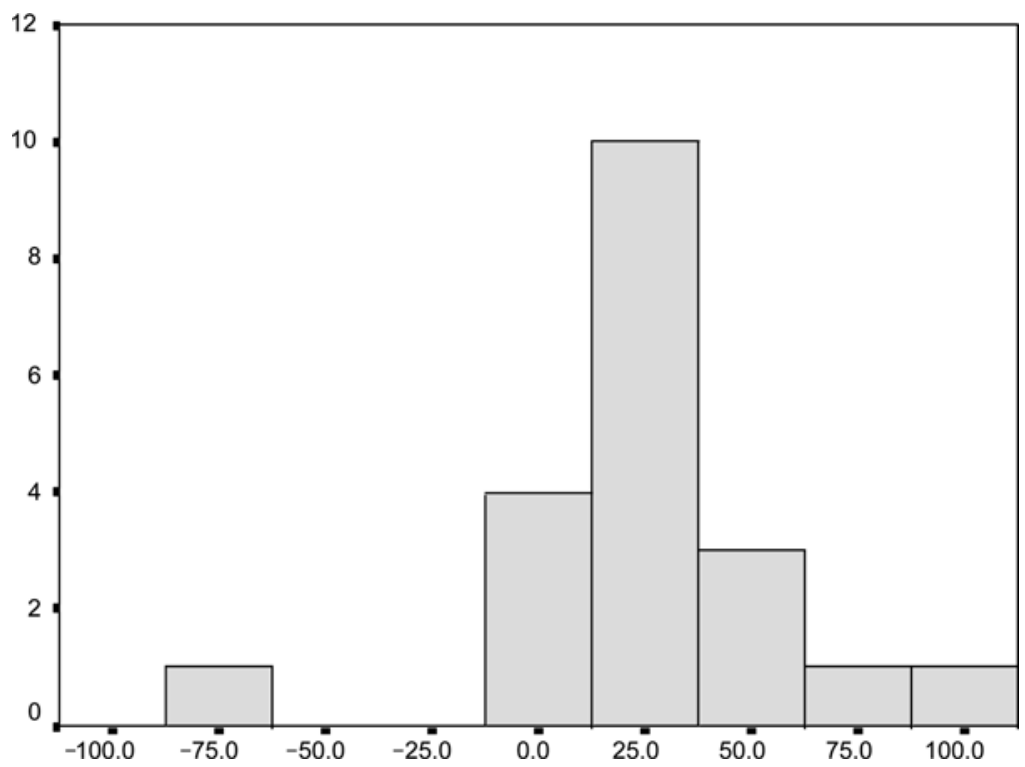

Figure 3 High intermediate learners $(n=20)$ : Percentage 'yes' responses to discontinuous answers in different-object contexts minus percentage 'yes' responses to discontinuous answers in same-object contexts

Notes: Difference: accepted discontinuous answers in different vs. same-object contexts; $\mathrm{SD}=33.07 ;$ Mean $=18.8$.

In support of the view that these implicatures arise in entailmentbased scales, we administered a second experiment in which we investigated the interaction of discontinuous quantifiers with the universal quantifier toujours 'always' and the existential quantifier parfois 'sometimes'. In the target language, a sentence of the form $\mathrm{Q}_{\exists}$... parfois ... [restriction of $\mathrm{Q}_{\exists}$ ] is potentially associated with either scenario. However, a sentence of the form $\mathrm{Q}_{\exists}$... toujours ... [restriction of $\mathrm{Q}_{\exists}$ ] pragmatically implies a different object per event. In Experiment 2, discontinuous quantifiers with toujours and parfois were crossed with contexts involving a different object per event and the same object for all events. Forty-seven learners participated in this additional experiment.

As in Experiment 1, each item in the experimental instrument included a story told in English by a character Mrs Briggs. The story provided either a scenario where there was a different object per event or the same theme for all events. This was followed by a request for 
information about the story, (Qu'est-ce vous avez donc appris? 'So, what did you learn?') from a French-speaking character Madame Goyette, and a discontinuous answer in response to the information request containing either parfois 'sometimes' or toujours 'always'. Respondents were to indicate whether the answer was an adequate response to the information query. Experimental items consisted of eight sets of quadruples. For each quadruple, there was one prose narrative unambiguously involving the same object and another unambiguously involving a different object per event. Each was paired once with a sentence exemplifying a discontinuous answer containing parfois and once with a discontinuous answer containing toujours. An example of a test item follows:

Sample Test Item 3 (different object per event with parfois):

Mrs Briggs: On three of the five voyages to the planet Omega taken so far, the mission's field scientist observed a truly remarkable thing in the universe: On the first mission, an immense geyser was observed throwing liquid nitrogen twenty miles high into the atmosphere of the planet. On the second mission, an eruption of the universe's largest known volcano was observed. On the third mission, an earthquake over the entire planet was observed. The last two missions however observed nothing of value. Still, NASA wants to organize more missions.

Mme Goyette: Qu'est-ce que vous avez donc appris?

Élève:

Sur la planète Oméga, quelque chose a parfois été observé de remarquable.

IS THIS AN ADEQUATE RESPONSE? YES NO CANNOT DECIDE

Eighteen fillers were blended with the rest of the task to offset the expected imbalance between the positive and negative responses. There was thus a total of 50 items presented in three distinct random orders.

An independent samples $t$-test comparing the relative acceptance of discontinuous answers with parfois 'sometimes' vs. discontinuous answers with toujours 'always' in same-object contexts shows that the two groups are indeed statistically distinct $(t(47)=2.52, p=.015)$. Low/mid intermediate learners $(n=28)$ showed evidence of sensitivity to scope interactions on this task, as shown by the asymmetry in Table 5. Thus, for (discontinuous) answers with parfois, there was no significant difference in respondents' rates of acceptance having a different object per event versus the same object per event $(t(27)=.87$, $p=.391$ ), but, crucially, for (discontinuous) answers with toujours, 
Table 5 Low/mid intermediate learners $(n=28)$ : percentage 'yes' on parfois vs. toujours discontinuous answers

\begin{tabular}{llllll}
\hline & \multicolumn{3}{c}{ parfois } & & \multicolumn{2}{c}{ toujours } \\
\cline { 2 - 3 } & Mean (\%) & SD & & Mean (\%) & SD \\
\hline Different objects & 75.00 & 17.68 & & 79.01 & 19.26 \\
The same object & 71.83 & 20.39 & & 64.73 & 21.53 \\
\hline
\end{tabular}

the acceptance rate in different-object contexts was significantly greater than the acceptance rate in same-object contexts $(t(27)=3.88$, $p=.001)$. This asymmetry is revealing of the role of the quantifier in the derivation of these implicatures. Although the acceptance rate for (discontinuous) answers is lower with toujours than with parfois in same-object contexts, this does not reach statistical significance.

In the case of high intermediate learners (Table 6), the lowest acceptance rate is still associated with answers with toujours in single-object contexts, although the results were not statistically significant. It may be that for (some of) these subjects, the number of events provided in the contexts (three) was not enough for them to consistently accept an answer with toujours. The existence of a response strategy relying on this aspect of context would then obfuscate any difference between different-object contexts and single-object contexts arising from an implicature.

In pursuance of this hypothesis, we considered the behaviour of those high intermediate learners (Group A, $n=8$ ) who accepted toujours in different-object contexts at a rate at least as high as the mean rate of acceptance of parfois in different-object contexts for the whole high intermediate population $(84.21 \%)$. This was compared to those high intermediate learners (Group B, $n=11$ ) who accepted toujours in

Table 6 High intermediate learners $(n=19)$ : percentage 'yes' on parfois vs. toujours discontinuous answers

\begin{tabular}{llllll}
\hline & \multicolumn{2}{c}{ parfois } & & \multicolumn{2}{c}{ toujours } \\
\cline { 2 - 3 } & Mean (\%) & SD & & Mean (\%) & SD \\
\hline Different objects & 84.21 & 18.09 & & 78.29 & 13.72 \\
The same object & 82.63 & 15.67 & & 76.45 & 10.32 \\
\hline
\end{tabular}


different-object contexts at a rate lower than the mean rate of acceptance of parfois in different-object contexts. Thus, Group B learners were those who seemed hesitant to use toujours for only three events, whereas Group A learners allowed the use of toujours at least as often as parfois in different object contexts.

For Group A learners, the mean acceptance rate for (discontinuous) answers with toujours in different-object contexts (92.19\%) was not significantly different from the mean acceptance rate for (discontinuous) answers with parfois in different-object contexts $(82.81 \%)(t(7)=$ $1.34, p=.222$ ). However, the acceptance of (discontinuous) answers with toujours $(75.31 \%)$ versus parfois $(88.44 \%)$ in same-object contexts contrasted significantly $(t(7)=4.11, p=.005)$. Furthermore, Group A learners' acceptance rates for answers with parfois in different-object contexts $(82.81 \%)$ and in same-object contexts $(88.44 \%)$ did not differ significantly $(t(7)=1.15, p=.287)$. However, their acceptance of answers with toujours in different-object contexts $(92.19 \%)$ was significantly greater than in same-object contexts $(75.31 \%)(t(7)=$ $4.22, p=.004)$. This suggests that a theoretically significant asymmetry appeared between parfois and toujours answers in different-object versus same object-contexts. Thus, Group A learners' acceptance patterns seem underlain by conversational conclusions that are scope induced and entailment based.

For Group B learners, the mean acceptance rate for (discontinuous) answers with toujours in different-object context (58.18\%) was significantly different from their acceptance rate for (discontinuous) answers with parfois in different-object contexts $(85.23 \%)(t(10)=3.16$, $p=.010)$. It indeed seems to be the case that Group B learners were reluctant to accept the use of toujours as a characterization of contexts with only three events. There was no contrast between parfois $(78.41 \%)$ and toujours $(77.27 \%)$ in same-object contexts $(t(10)=.20, p=.849)$. No contrast arose between (discontinuous) answers with parfois in different-object contexts $(85.23 \%)$ versus same-object contexts $(78.41 \%)(t(10)=1.26, p=.237)$. But, a contrast in the opposite direction was found with toujours: Group B learners' acceptance of answers with toujours in different-object contexts $(68.18 \%)$ was significantly lower than their acceptance of answers with toujours in same-object contexts $(77.27 \%)(t(10)=3.29, p=.008)$. 
In summary, the response pattern of Group A learners seems underlain by implicatures that are derived in entailment-derived informational scales at the output of syntax. In contrast, Group B results follow if aspects of the context constitute the central ingredient of the response pattern. Specifically, three events did not suffice for confident use of toujours in characterizing the scenario, but salience of the same theme for all three events improved the use of toujours in the characterization of what happened. This contrast between Group A and Group B respondents suggests to us that the relevant features of the target language allowing discontinuous quantifiers are not acquired by Group B respondents, so that the interpretive consequences of discontinuous quantifiers were not felt.

That there is a difference in the acquisition of discontinuous quantifiers between Groups A and B finds confirmation in a further analysis of results in Experiment $1 .^{2}$ Group A respondents evinced the interpretive knowledge associated with the grammaticality of discontinuous quantifiers: they accepted continuous answers (87.50\%) and discontinuous answers $(85.72 \%)$ at similar rates in different-object contexts $(t(6)=.13$, $p=.899)$. Crucially, their acceptance of continuous answers $(87.50 \%)$ differed significantly from their acceptance of discontinuous answers $(62.50 \%)$ in same-object contexts $(t(6)=3.06, p=.022)$. Again, Group A learners' acceptance of continuous answers in different-object contexts $(87.50 \%)$ and their acceptance of continuous answers in sameobject contexts $(87.50 \%)$ were totally flat $(t(6)=.00, p=1)$, but again, crucially, their acceptance of discontinuous answers in different-object contexts $(85.71 \%)$ and in same-object contexts $(62.50 \%)$ contrasted significantly $(t(6)=2.77, p=.032)$. These results are consonant with the interpretive consequences of a grammar licensing discontinuous quantifiers at the syntax-semantics-pragmatics interfaces.

In contradistinction, the results of Group B respondents on Experiment 1 did not reveal evidence of French-like interpretive knowledge. These learners accepted continuous answers $(81.82 \%)$ and discontinuous answers $(85.23 \%)$ at similar rates in both different-object contexts $(t(10)=.56, p=.588)$ and same-object contexts: $77.27 \%$ (continuous)

${ }^{2}$ One respondent from Group A and two respondents from Group B did not participate in Experiment 1. This is reflected in the degree of freedom values of the $t$-tests performed on results of Experiment 1 for these two groups. 
and $69.32 \%$ (discontinuous) $(t(10)=1.05, p=.319)$. Their rates for continuous answers in different-object contexts $(81.82 \%)$ versus same-object contexts $(77.27 \%)$ are not statistically different $(t(10)=.36, p=.727)$; and neither are their acceptance rates for discontinuous answers in different-object contexts $(85.23 \%)$ versus same-object contexts $(69.32 \%)$ $(t(10)=1.26, p=.236)$.

An independent samples $t$-test comparing Group A respondents and Group B respondents in terms of their relative acceptance of continuous versus discontinuous answers in same-object contexts provides statistical confirmation that Group A and Group B respondents indeed exhibit distinct response patterns in Experiment $1(t(16)=2.30, p=.035)$.

The French native speakers' results were flat on this task, with all differences far from achieving significance (see Table 7). All sentences on this task are stylistically disfavoured. It is possible that stylistic concerns may have rendered the task more difficult for native speakers. As a more general point, Rex A. Sprouse (personal communication) pointed out to us that for some asymmetry not to arise in a native comparison group in contrast to some nonnative group does not absolve the researcher from exploring the range of possible models for the asymmetry found in second language (L2) acquisition. We agree with him. The fact that a theoretically relevant asymmetry arose in an L2 group cannot be overlooked because logically such an asymmetry reveals that an inference is necessarily made that goes beyond the literal semantics, and that this inference is prima facie sensitive to entailment relations between scope-dependent semantic representations. The fact that L2 learners may exhibit theoretically relevant knowledge that is obfuscated in some other group is a distinct possibility that should not be overlooked.

Table 7 Native speakers $(n=16)$ : percentage 'yes' on parfois vs. toujours discontinuous answers

\begin{tabular}{llllll}
\hline & \multicolumn{2}{c}{ parfois } & & \multicolumn{2}{c}{ toujours } \\
\cline { 2 - 3 } \cline { 6 - 6 } & Mean (\%) & SD & & Mean (\%) & SD \\
\hline Different objects & 80.47 & 22.81 & & 79.75 & 20.34 \\
The same object & 73.44 & 17.00 & & 75.00 & 14.43 \\
\hline
\end{tabular}


Thus, the very existence of these asymmetries in English-French interpretive knowledge suggests that discontinuous structures receive $\forall \exists$ representations at LF, while the continuous structure allows both $\forall \exists$ and $\exists \forall$ LF representations, and that $\forall \exists$ LF representations trigger the implicature "and the $\exists \forall$ representation is not verified". These conclusions are based on the fact that asymmetries are not predicted from the range of possible LF representations without the constraint in (8) and without implicatures supervening on $\forall \exists$ LF representations. If implicatures were not required, no asymmetry would arise in a normal sample. The asymmetries, therefore, reveal both domain-specific constraints on LF representations and mandatory implicatures supervening on $\forall \exists$ LF representations. The results from Experiment 2 support the role of semantic scales in constraining the manner in which the implicatures are computed.

Interlanguage asymmetries that follow from constraint (8) and the derivation of LF-triggered and semantically calculated implicatures provide prima facie evidence for a domain-specific, informationally encapsulated algorithm constraining L2 acquisition. We now show that the effects of constraint (8) derive from general principles of movement, so that it never has to be acquired. We then show that only narrowly constrained semantic computations satisfying principles of cooperation in idiosyncratic ways guarantee the appearance of these asymmetries.

\section{A domain-specific, informationally encapsulated processor}

\section{Domain specificity}

Assuming with Chomsky (1995) that movement (conceived as copying followed by matching deletions) is driven by the need to satisfy the checking of features and by output requirements, we show that the constraint on the legitimate semantic representations available to continuous and discontinuous quantifiers seems to be a reflex of the theory of movements. We assume that movement to the subject position is driven by the need to satisfy the Extended Projection Principle (EPP) requirements of I, with nominative case a reflex of this satisfaction. Following Hulk and Verheugd (1994), we assume that a quantificational [+Q] feature of the de morphology licenses a null [+Q] category in its specifier position, 
which allows for discontinuous quantification. Thus, the feature requirements of I (i.e., EPP, and Case) can satisfied by the DP quelque chose de remarquable as in (12) or the DP quelque chose as in (13).

12) [ [IP Quelque chose de remarquable a [VP été observé [ $\left.{ }_{\mathrm{DP}} t_{\text {quelque chose de remarquable }}\right]$ par chacun des chercheurs]

13) [IP Quelque chose a [VP été observé $\left[_{\mathrm{DP}} t_{\text {quelque chose }}\right.$ [de remarquable ]]] par chacun des chercheurs ]

We assume with Diesing (1992) that indefinite expressions such as quelque chose are ambiguous between a quantificational interpretation (associated with the IP domain) that possesses inherent existential force lexically, and an individual variable interpretation (associated with the VP domain) with its existential force provided constructionally by a default Existential Closure operation at the VP-level. The by-phrase introduced by par is treated as an inherently case-marked (VP-external) syntactic adjunct binding the external argument position of the verb. The by-phrase takes scope over the VP-level existential closure but takes scope under the IP-level quantifier.

The derivations in (12) and (13) interact with deep properties of grammar to determine mappings to semantic representations. Matching deletions in the head or tail position in (12) lead to two possible LF representations: (14) and (15). In (14), the indefinite is interpreted at the foot of the chain and takes scope under the by phrase. In (15), the indefinite is interpreted at the head of the chain and takes scope over the by phrase.

14) $\left[{ }_{\mathrm{VP}} \forall \mathrm{y}[\operatorname{researcher}(\mathrm{y}) \rightarrow \exists \mathrm{x}[\mathrm{VP} \operatorname{observe}(\mathrm{x})(\mathrm{y}) \wedge\right.$ thing $\left.(\mathrm{x}) \wedge \operatorname{remarkable}(\mathrm{x})]]\right]$

15) $\left[{ }_{I P} \exists x\left[\operatorname{thing}(x) \wedge \operatorname{remarkable}(x) \wedge\left[{ }_{V P} \forall y\left[{ }_{V P} \operatorname{researcher}(y) \rightarrow \operatorname{observe}(\mathrm{x})(\mathrm{y})\right]\right]\right]\right]$

Crucially, once the DP quelque chose is raised to SpecI as in the derivation in (13), the feature-driven movement of the expression [t(quelque chose) de remarquable] out of the VP to SpecI is ruled out by Last Resort (which says that an operation cannot happen unless it is required) because the relevant formal features of I (EPP and case) are already satisfied. Movement for interpretive reasons (i.e., scope taking) is also ruled out, since the trace in the raised DP is not head-governed (Lamontagne and Travis, 1987). Thus, if quelque chose is raised, further movements of [t(quelque chose) de remarquable] are ruled out. If quelque chose de remarquable is raised to SpecI, then Full 
Interpretation at the Phonological Form (PF) interface requires that the entire expression quelque chose de remarquable be uttered.

In summary, if the expression quelque chose is raised, the indefinite quelque chose de remarquable is uniquely interpreted within the VP. However, if the whole expression quelque chose de remarquable is raised, the indefinite quelque chose de remarquable may be interpreted either in IP or at the trace. The range of legitimate LF representations need not be acquired, but inheres in a specific grammatical organization.

\section{Informational encapsulation}

We now consider that, instead of computations through scales (our position), conversational implications derived on the basis of beliefs might be the source of the asymmetries found in English-French interlanguage. We show that this cannot feasibly be. Thus, in Experiment 1, learners' behaviour would be due to conversational implications derived on the basis of beliefs about events with human actors. Hence, learners would (oddly) share the belief that real world observations (by distinct scientists) typically do not have the same theme. In accordance with this belief, they would exclude from literal interpretations the states of affairs in which all relevant events are observations of the same object. The reasoning would be as follows:

Semantics: For every researcher there is a remarkable thing such that he or she observed it. World Belief 1: Different things get observed by distinct scientists.

Conversational conclusion: The observations were not of the same thing.

Such beliefs about events with human actors might be compatible with the learner behaviour observed in Experiment 1, but only if these beliefs were generally held and generally felt to be relevant to the situation of utterance. However, in this latter respect, the information in sameobject scenarios should tell the learner that the current situation is not prototypical, but rather an exception to the belief. Once the belief is irrelevant, the conversational conclusion can no longer be drawn. Hence, no asymmetry should appear.

If beliefs about events determined conversational conclusions, then additionally scope-determined entailment relations would be irrelevant. Therefore, the asymmetry observed in Experiment 2 between the 
existential quantifier parfois 'sometimes' versus the universal quantifier toujours 'always' would be unexplained. To see that the same expectation for both toujours 'always' and parfois 'sometimes' would hold in the belief-based model, we consider a corollary world belief about scientific observations: that different things get observed on different occasions. World Belief 2 would license a conversational conclusion that excludes the states of affairs in which all relevant observations are of the same object:

Semantics: Sometimes/Always a remarkable thing was observed.

World Belief 2: Different things get observed on different occasions.

Conversational conclusion: The observations were not of the same thing.

This inference would arise whether the quantifier is parfois 'sometimes' or toujours 'always' because the quantifier scope is irrelevant; only the belief about certain kinds of events matters. In this case, a conversational conclusion would be derived with both parfois 'sometimes' and toujours 'always'. Therefore, acceptance rates in same-object contexts would be significantly depressed with both parfois 'sometimes' or toujours 'always'. No such pattern was found.

In summation, the pragmatic inferences that account for the asymmetries observed in the development of English-French interpretive knowledge do not appear to follow from a belief-dependent strengthening of the informational content of utterances beyond the literal semantics. Rather, these pragmatic inferences in interlanguage interpretation seem to be grammatically triggered and calculated (Kadmon, 1987; 2000; Horn, 1992; Landman, 1998). Such scalar implicatures follow from general principles of conversational co-operation, but in a domain-specific and informationally encapsulated manner. Hence, the scale-dependent strengthening of literal meanings evident in English-French acquisition of discontinuous quantifiers strongly suggests that aspects of L2 interpretive knowledge are reflexes of an idiosyncratic interface between a specialized language module and general principles of co-operative behaviour in the type of mental architecture explored in Fodor $(1983 ; 2000)$.

\section{The source of language specific computations}

Given that the range of legitimate mappings to semantic representations derives from the theory of movements, the knowledge accounting for 
the interpretation of discontinuous quantifiers is naturally instantiated in English, although discontinuous quantifiers of the type found in French are not. As an anonymous Second Language Research reviewer notes, the English sentence Each of the researchers observed a remarkable thing strongly suggests that it was not the same thing that was observed. This intuition also follows from an LF-derived implicature through scales. Thus, one may wonder whether the asymmetries in English-French interpretation reflect a (largely) universal computational module that interacts with the functional lexicon (as investigated above), or whether they can be simply derived from the L1 knowledge, without the need for a universal algorithm (surviving in the adult mind) (Schachter, 1989; Bley-Vroman, 1990). As Hale (1996) points out, given the level of generality of principles of Universal Grammar in Minimalist understanding, all grammatical principles are instantiated in all languages. The distinction becomes vitiated since knowledge of the L1 is the product of a parameterized lexicon with general universal principles.

However, the performance of L2 learners on the task might plausibly be due to L1 intuitions, not requiring any learning. Thus, following Dekydtspotter et al. (1997), we consider the intuitions that knowledge of English might provide to learners, despite the ungrammaticality of partitive quantifiers with detachable restrictions. In conditions similar to the English-French learner groups, we tested two groups of native English respondents, presenting them with stimuli consisting of continuous and discontinuous sentences. These individuals were selected from first semester French college classes and had no or exceedingly little prior exposure to French.

They were told that they were judges for an (imaginary) final comprehension exercise in Madame Goyette's class populated with French speaking learners of English. As judges, respondents were presented with excerpts from the transcripts of the comprehension exercise consisting of a story told in English and the question "So, what happened?" followed by a pupil's response. Respondents were to indicate whether the answer was an adequate response to the information query. An answer was adequate as long as it did not mislead the hearer about the facts. As this was a comprehension exercise, respondents were told that "the potentially faulty grammar of a pupil's response must not be held 
Table 8 English controls $(n=14)$ : percentage 'yes' on continuous vs. discontinuous answers (without of)

\begin{tabular}{llllll}
\hline & \multicolumn{2}{l}{ Continuous answers } & & \multicolumn{2}{c}{ Discontinuous answers } \\
\cline { 2 - 3 } & Mean (\%) & SD & & Mean (\%) & SD \\
\hline Different objects & 91.07 & 19.87 & & 78.57 & 33.05 \\
The same object & 66.07 & 29.59 & & 70.54 & 27.56 \\
\hline
\end{tabular}

against the pupil." Respondents were thus to focus on the informational content conveyed by the answer of the pupil.

One English control group $(n=14)$ was presented with items as in "Something remarkable was observed by each of the researchers" and "Something was observed remarkable by each of the researchers" that gloss the French items, pace the de morphology. The other control group $(n=10)$ was presented with sentences as in "Something of remarkable was observed by each of the researchers" and "Something was observed of remarkable by each of the researchers" that gloss the French items verbatim. Tables 8 and 9 respectively show similar results for both groups.

In the first task (without of), English controls accepted continuous and discontinuous answers with scenarios involving a different object per participant at $91.07 \%$ and $78.57 \%$ respectively $(t(13)=1.49$, $p=.160)$. They also accepted continuous and discontinuous answers in the same object condition at $66.07 \%$ and $70.54 \%$ respectively $(t(13)=$ $.59, p=.567)$. This shows a response bias against accepting answers with scenarios involving the same object for all human participants. Contrasts of means were significant for continuous answers $(t(13)=$ $2.55, p=.024$ ). In the second task (with of ), continuous and discontinuous answers with scenarios involving a different object per participant were accepted at $85.00 \%$ and $77.50 \%$ respectively $(t(9)=.80, p=.443)$.

Table 9 English controls $(n=10)$ : percentage 'yes' on continuous vs. discontinuous answers (with of)

\begin{tabular}{llllll}
\hline & \multicolumn{2}{l}{ Continuous answers } & & \multicolumn{2}{c}{ Discontinuous answers } \\
\cline { 2 - 3 } & Mean (\%) & SD & & Mean (\%) & SD \\
\hline Different objects & 85.00 & 21.08 & & 77.50 & 28.14 \\
The same object & 55.00 & 23.72 & & 56.25 & 30.76 \\
\hline
\end{tabular}


Continuous and discontinuous answers were accepted in same object contexts at $55.00 \%$ and $56.25 \%$ respectively $(t(9)=.23, p=.823)$. English respondents seemed again biased against accepting the answers with scenarios involving the same object for all human participants. Significant contrasts of means appeared for continuous answers $(t(9)=$ 2.84, $p=.019)$.

The behaviour of English controls was reminiscent of the low $/ \mathrm{mid}$ intermediate learners, who showed similar rates of acceptance between continuous and discontinuous answers in same-object contexts and similar rates of acceptance between continuous and discontinuous answers in different-object contexts, with a general bias against same-object interpretations. The results of both control groups and the low/mid intermediate group were compatible with the ungrammaticality of leftbranch extractions: the effects of a (French-like) grammar are simply not felt on interpretation for these groups. This was in stark contrast with the French-like behaviour of high intermediate learners who accepted continuous and discontinuous answers in different-object contexts equally, but accepted continuous answers significantly more than discontinuous answers in same-object contexts.

Hence, English intuitions do not explain the emergence of these asymmetries in English-French acquisition. Rather, we argue that the acquisition of the morphology allowing left-branch extractions in a grammar determines the appearance of the interpretive effects in the strict constraints of the interfaces between syntax, semantics and pragmatics. Our findings, therefore, provide strong support for Schwartz's (1986; 1987; 1999) claim that L2 acquisition is constrained by a (largely) universal processor devoted to language, of which Universal Grammar specifies the contents.

\section{Conclusions}

The interpretation of quantifiers with detachable restrictions in English-French interlanguage shows reflexes of domain specificity in the limits on available LF representations. It also shows reflexes of informational encapsulation in computations at the interface between grammar and principles of conversational cooperation. The interpretation of (dis)continuous quantifiers in English-French interlanguage 
reveals asymmetries that - given the truth-conditional relations between possible LF representations - require a constraint on the LF representations available to discontinuous constituents and also require semantically constrained pragmatic computations supervening on specific LF representations. These properties of knowledge acquired in EnglishFrench acquisition follow naturally if L2 acquisition is constrained by an encapsulated processor with Universal Grammar for its database, as Schwartz (1999) entertains. Such properties are otherwise unexpected if a range of computations are available in L2 acquisition.

\section{Acknowledgements}

This work was funded in part by a Faculty Graduate Research Fund grant from Indiana University administered through the Indiana University Department of French and Italian. We acknowledge the comments of three anonymous Second Language Research reviewers which helped us substantially refine our analysis. We thank Rex A. Sprouse for methodological input during the development stage and Bonnie D. Schwartz for criticism during the drafting stage. We benefited from feedback from audiences during a poster presentation at Generative Approaches to Second Language Acquisition, 6, Université d'Ottawa and from members of the Indiana University second language research group. The final presentation also owes much to Roger Hawkins' editorial advice. Any remaining technical and rhetorical inadequacies are our sole responsibility. Lastly, we would like to thank the subjects, instructors and course administrators for their co-operation. In particular, we acknowledge the help of Kathleen O'Connor with the collection of data from native French respondents in Lille.

\section{References}

Azoulay-Vicente, A. 1985: Les tours comportant l'expression de + adjectif [Turns of phrase including de + adjective]. Geneva: Librairie Droz.

Birdsong, D. 1985: Constraints on the structure de + modifier in French. In King, L. and Maley, C.A., editors, Selected papers from the 13th linguistics symposium on romance languages. Philadelphia, PA: John Benjamins. Bley-Vroman, R. 1990: The logical problem of second language learning. Linguistic Analysis 20, 3-49. 
Chomsky, N. 1995: The minimalist program. Cambridge, MA: MIT Press.

Dekydtspotter, L., Sprouse, R.A. and Anderson, B. 1997: The interpretive interface in L2 acquisition: the process-result distinction in EnglishFrench interlanguage grammars. Language Acquisition 6, 297-332.

de Swart, H. 1992: Intervention effects, monotonicity and scope. In Barker, C. and Dowty, D., editors, Proceedings of the second conference on semantics and linguistic theory. Columbus, $\mathrm{OH}$ : Ohio State University, Department of Linguistics.

Diesing, M. 1992: Indefinites. Cambridge, MA: MIT Press.

Fodor, J.A. 1983: The modularity of mind. Cambridge, MA: MIT Press. 2000: The mind does not work that way. Cambridge, MA: MIT Press.

Grice, H.P. 1975: Logic and conversation. In Cole, P. and Morgan, J., editors, Speech acts. Syntax and semantics. Volume 3. New York: Academic Press. Hale, K. 1996: Can UG and L1 be distinguished in L2 acquisition? Behavioural and Brain Sciences 19, 726-28.

Horn, L.R. 1985: Metalinguistic negation and pragmatic ambiguity. Language 61, 121-74.

1989: A natural history of negation. Chicago, IL: The University of Chicago Press.

1992: The said and the unsaid. In Barker, C. and Dowty, D., editors, Proceedings of the second conference on semantics and linguistic theory. Columbus, $\mathrm{OH}$ : Ohio State University, Department of Linguistics.

Hulk, A. and Verheugd, E. 1994: Accord et opérateurs nuls dans les projections adjectivales [Agreement and null operators in adjectival projections]. Revue québécoise de linguistique 23, 17-45.

Huot, H. 1981: Constructions infinitives du français: le subordonnant 'de' [French infinitival constructions: the subordinator de]. Geneva: Librairie Droz.

Kadmon, N. 1987: On unique and non-unique reference and asymmetric quantification. New York: Garland.

2000: Formal pragmatics: semantics, pragmatics, presupposition, and focus. Malden, MA: Blackwell.

Lamontagne, G. and Travis, L. 1987: The syntax of adjacency. In Crowehurst, M., editor, Proceedings of the West Coast conference on formal linguistics, 6. Stanford, CA: Stanford Linguistics Association, Stanford University, 173-85.

Landman, F. 1998: Plurals and maximalization. In Rothstein, S., editor, Events and grammar. Dordrecht: Kluwer Academics.

Magnan, S. 1986: Accessing speaking proficiency in the undergraduate curriculum: data from French. Foreign Language Annals 5, 429-38.

Schachter, J. 1989: Testing a proposed universal. In Gass, S. and Schachter, J., editors, Linguistic perspectives on second language acquisition. New York: Cambridge University Press. 
Schwartz, B.D. 1986: The epistemological status of second language acquisition. Second Language Research 2, 120-59.

— 1987: The modular basis of second language acquisition. Unpublished doctoral dissertation, University of Southern California, Los Angeles, CA.

1999: Let's make up your mind: 'special nativist' perspectives on language, modularity of mind, and nonnative language acquisition. Studies in Second Language Acquisition 22, 635-55.

\section{Appendix 1 Test sentences}

1 con. Quelque chose - de remarquable - a été observé par chacun des scientifiques.

1 dis. Quelque chose a été observé - de remarquable - par chacun des scientifiques.

2 con. Quelque chose - de très traditionnel - a été selectionné par chacun des architectes.

2 dis. Quelque chose a été selectionné - de très traditionnel - par chacun des architectes.

3 con. Quelque chose - de choquant - a été proposé par chacun des artistes.

3 dis. Quelque chose a été proposé - de choquant - par chacun des artistes.

4 con. Quelque chose - de terrifiant - a été choisi par chacun des étudiants.

4 dis. Quelque chose a été choisi - de terrifiant - par chacun des étudiants.

5 con. Quelque chose - de lamentable - a été vu par chacun des enfants.

5 dis. Quelque chose a été vu - de lamentable - par chacun des enfants.

6 con. Quelque chose - de fabuleux - a été découvert par chacun des voleurs.

6 dis. Quelque chose a été découvert - de fabuleux - par chacun des voleurs.

7 con. Quelque chose - de formidable - a été trouvé par chacun des paléontologues. 
7 dis. Quelque chose a été trouvé - de formidable - par chacun des paléontologues.

8 con. Quelque chose - de fantastique - a été démontré par chacun de ces savants.

8 dis. Quelque chose a été démontré - de fantastique - par chacun de ces savants. 